\title{
Design, simulation, and quality evaluation of micro-optical freeform beam shapers at different illumination conditions
}

\author{
Daniel Infante-Gómez ${ }^{1,2, *}$ and hans Peter Herzig ${ }^{1}$ \\ ${ }^{1}$ Optics \& Photonics Technology Laboratory, École Polytechnique Fédérale de Lausanne (EPFL), Rue de la Maladière 71, CH-2000 Neuchâtel, Switzerland \\ ${ }^{2}$ Laboratory for Advanced Materials Processing, Swiss Federal Laboratories for Materials Science and Technology (EMPA), Feuerwerkerstrasse 39, CH-3603 \\ Thun, Switzerland \\ *Corresponding author: daniel.infante@epfl.ch
}

Received 24 May 2016; revised 8 September 2016; accepted 10 September 2016; posted 12 September 2016 (Doc. ID 266894); published 10 October 2016

\begin{abstract}
A design method to generate thin micro-optical freeform (MOF) beam shapers by clipping or wrapping an original and much thicker freeform surface is provided. MOF elements are situated at the border between refractive and diffractive optical elements. The influence of parameters such as the clipping factor $q$, the peak-to-valley amplitude of the original surface, the design wavelength and the spectrum of the light source (single wavelength and multiple wavelength lines) into the quality of the output intensity distributions has been studied. Integer $q$ values are mandatory for good quality at monochromatic illumination. On the contrary, the quality obtained by broadband illumination oscillates with $q$ and peaks maximally at around $q=3$. () 2016 Optical Society of America
\end{abstract}

OCIS codes: (050.1970) Diffractive optics; (220.0220) Optical design and fabrication; (350.3950) Micro-optics.

http://dx.doi.org/10.1364/AO.55.008340

\section{INTRODUCTION}

Advances in optics have been closely related to progress in manufacturing methods. As an example, the arrival of powerful and fast computers as well as the development made in computer numerical control (CNC) tools were extremely important factors in the design and fabrication of complex optical elements, such as aspherical and freeform surfaces[1,2]. In a similar way, laser sources paved the way to diffractive optical elements (DOE), which have experienced a strong interest from the optics research community in the last 25 years [3].

Transmissive optical elements can be mainly classified into two divisions or domains: refractive and diffractive. Aspherical and freeform surfaces belong to the former domain, whereas DOEs fit in the later division. In the following paragraphs, a comparison of the two domains is made in order to emphasize the differences among them.

Ray-tracing techniques are sufficient to explain light propagation through and beyond refractive elements. On the other hand, since interferences are the basis of the diffractive domain, the wave behavior of light must be contemplated when designing diffractive elements.

Differences are also significant when comparing the topography of elements from each domain. The refractive domain is characterized by surfaces with macroscopic continuous features which are mostly insensitive to the wavelength of light: only material dispersion plays a role in transmissive refractive elements. Conversely, the DOEs wavelength dependence is prominent, since they are thin elements with microscopic features that modulate incident light by means of diffraction. These devices require highly coherent light sources for proper operation and are very sensitive to variations in the illumination wavelength and to fabrication errors. Furthermore, the quality of the intensity distributions they produce is substantially reduced by the effect of speckle noise [4].

Caustic generators are examples of refractive beam-shaping freeform surfaces [5,6]. Their surface shapes are obtained after inputting the target intensity distribution into an algorithm that solves the inverse problem by geometric propagation of light rays. On the other hand, the iterative Fourier transform algorithm (IFTA) is one of the most widely used methods in the design and optimization of DOEs [7-9].

Numerous studies on hybrid optical elements, i.e. elements combining both refractive and diffractive properties, have been made in the last decades [10-13]. The need for mastering beamshaping techniques is continuously increasing in industries such as consumer electronics, illumination, biomedical engineering, defense and security [14-18]. In this context, elements combining the advantages of both domains in terms of image quality, light source independence, thus wavelength insensitivity, and 
compactness, are solid candidates to meet the requirements set by industry.

The fabrication of refractive beam-shaping freeform elements is well mastered. Techniques like multi-axis ultra-precision diamond turning or micro-milling allow the fabrication of such non-rotationally symmetric structures with outstanding accuracy and resolution [2]. Despite the fact that surface finish can be excellent for these "macro"-structures, the machining process can be significantly time-consuming and inefficient, especially in the case of raster milling.

A miniaturization and/or flattening of freeform beamshaping structures is sought, since it will ensure compactness and hence better system integration. Nonetheless, attention must be paid to this miniaturization process, since it could not be infinitely performed. The étendue, i.e. the product of source area by the solid angle of a certain light beam, has to be conserved. A significant element thickness reduction, while keeping the projection distance constant and without impairing the optical performance, requires thus a different design strategy.

Tooling-related issues (tool path, tool orientation, and tool geometry) make CNC milling inappropriate for micro-optical freeform (MOF) structures. Laser writing lithography has been used to machine freeform structures with low peak-to-valley amplitudes. Higher peak-to-valley surfaces (up to $60 \mu \mathrm{m}$ ) can be achieved at the expense of surface quality and process repeatability, owing to the lack of control of photoresist dynamics [19]. High intensity light beam ablation, using an excimer laser, can address ablation depths as low as $100 \mathrm{~nm}$ on large and shallow areas, hence allowing the fabrication of MOF elements while keeping an acceptable surface quality $[20,21]$.

This work presents a top-down approach to design miniaturized beam-shaping structures lying at the border between diffractive and refractive domains, so-called MOF elements [22], and moreover studies the limits between the two domains. The limitations of the fabrication method are also considered in this design strategy. Eventually, an experimental result is presented.

\section{DESIGN METHOD}

MOF element design starts by the generation of a freeform beamshaping surface, obtained, for instance, by the method described in [6]. Depending on its dimensions, proper lateral and vertical scaling might be necessary to downsize the original surface and/or its projection distance. A further reduction in thickness of the element can be achieved by wrapping or clipping its surface profile, while keeping the image size and projection distance constant. This procedure is described by the following equations:

$$
\begin{gathered}
h_{c}(x, y)=h(x, y) \bmod H_{\mathcal{C}} \\
H_{c}=q \lambda_{\mathrm{D}} / \Delta n\left(\lambda_{\mathrm{D}}\right)
\end{gathered}
$$

where $q \subset \mathbb{R}_{>0}$ is the clipping factor, $\lambda_{\mathrm{D}}$ is the design wavelength, and $\Delta n(\lambda)$ is the refractive index difference between the element material and the surrounding medium. For the special case of $q=1$, the wrapping or clipping height $H_{c}$ corresponds to a $2 \pi$-phase shift of the output light field. The schematic on Figure 1 shows the typical arrangement for a MOF beam shaper.

Three freeform beam shapers have been studied in this work. They generate as output the portrait images of: Alan Turing (AT), Rafael Nadal (RN), and Roger Federer (RF). Table 1 shows the dimensions and other parameters of these elements: in-plane area $A$, peak-to-valley amplitude $P V$, projection distance $z$, sampling size $\Delta x$ and number of samples $M$ and $N$. The sampling size has been kept constant for both $x$ and $y$ axes as well as for both element and image planes. The intensity distribution generated by each element $I_{\text {out }}$ has also been included there. Note the disparity in contrast among the different $I_{\text {out }}$, which is directly related to the algorithm used in the generation of the freeform surface, to the different element dimensions ( $A$ and $P V$ values), and also to the gradients of the surfaces. For instance, the RF beam shaper, being flatter and slightly smaller than its counterparts, generates a lower contrast $I_{\text {out }}$. The design wavelengths used in the simulations, as well as the refractive index of the element material (polycarbonate surrounded by air), can be checked in Table 2 .

Table 1. Relevant Parameters for the Three Freeform Beam Shapers Used in This Work

\begin{tabular}{cccc}
\hline & AT & RN & RF \\
\hline$A\left[\mathrm{~mm}^{2}\right]$ & $6.40 \times 6.40$ & $6.41 \times 6.41$ & $6.15 \times 6.15$ \\
$P V[\mu \mathrm{m}]$ & 215.20 & 447.90 & 102.94 \\
$z[\mathrm{~mm}]$ & 6.40 & 17.00 & 17.00 \\
$\Delta x[\mu \mathrm{m}]$ & 0.32 & 0.32 & 0.31 \\
$M, N$ & 20000 & 20000 & 20000 \\
$I_{\text {out }}$ & & & \\
& &
\end{tabular}

Table 2. Clipping Height Values for Different Design Wavelengths.

\begin{tabular}{ccc}
\hline$\lambda_{\mathrm{D}}[\mathrm{nm}]$ & $n\left(\lambda_{\mathrm{D}}\right)$ & $H_{c}(q=1)[\mu \mathrm{m}]$ \\
\hline 532 & 1.5917 & 0.899 \\
641 & 1.5798 & 1.106 \\
\hline
\end{tabular}

It will be proved in the following sections that the clipping/wrapping method can significantly reduce the thickness of the MOF beam shaper, at the expense of introducing multiple folds or wrinkles in the otherwise highly continuous surface. Therefore, it exists a tradeoff between output image quality and vertical compactness of the beam shaper. For instance, for $q=10$ at $\lambda_{\mathrm{D}}=641 \mathrm{~nm}$ the final $P V$ amplitude is just $11.06 \mu \mathrm{m}, c a .5 .1 \%$ of the initial value for the AT element. On the one hand, attention must be paid so as to avoid violation of the Nyqvist sampling rate in the simulation of light propagation through the structures. On the other hand, from a fabrication point of view, clipped MOF structures are more challenging to be produced than standard and smooth freeform ones.

\section{SIMULATION RESULTS}

Free-space light propagation beyond the MOF elements has been simulated by means of the angular spectrum of planar waves method [23] in Matlab. The structures have been approximated as thin phase elements.

In order to both respect sampling constraints and consider fabrication inaccuracies of sharp edges into the simulations, the clipped height profile has been convoluted with a Gaussian filter. 


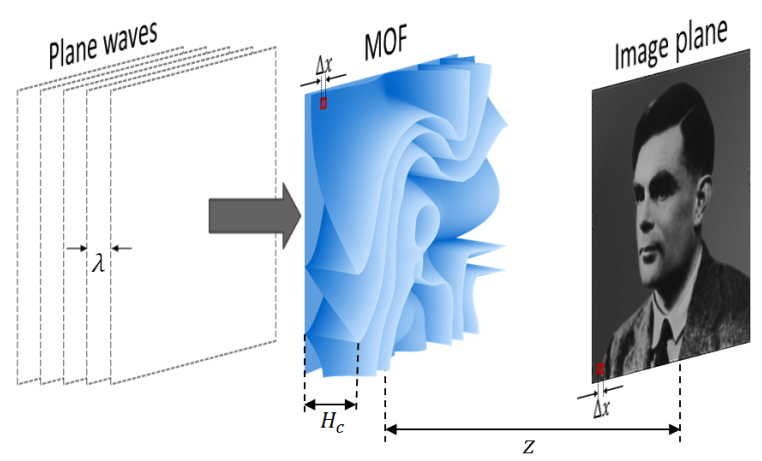

Fig. 1. Schematic showing the setup of a MOF beam shaper as well as some relevant parameters. The dimensions have been scaled for illustrative reasons.

Figure 2 plots the ratio of energy from the spectral power density (SPD) of the different clipped or wrapped height profiles lying inside the FWHM circle of the Gaussian filter SPD on the total energy. A FWHM value in the space domain of $3 \mu \mathrm{m}$ has been chosen for this purpose, based on the lateral resolution of an excimer laser ablation fabrication process. Owing to a limitation in computational memory, simulations have been restricted to $q \leq 5$. The influence of filtering becomes noticeable when small clipping factors, e.g. $q \lesssim 1$, are applied. Furthermore, a flatter original element, i.e. having lower $P V$ amplitude for a similar lateral size and hence less number of folds, is less sensitive to the influence of the filter (see the RF curve standing above the rest in Figure 2).

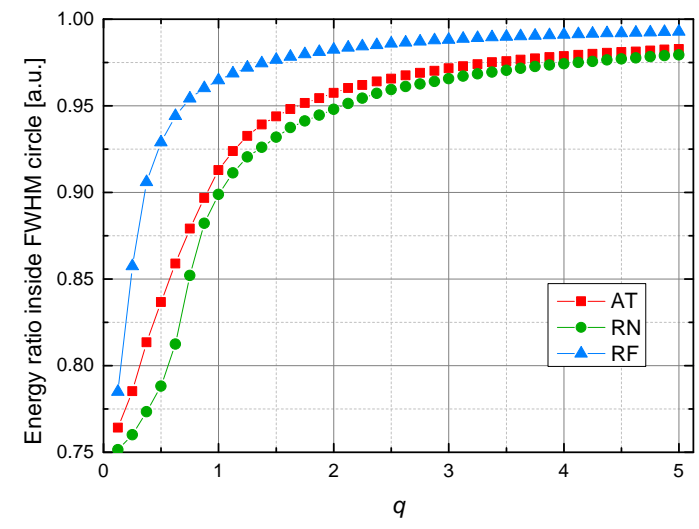

Fig. 2. Ratio of height profile spectral power density (SPD) lying inside the FWHM circle of the Gaussian filter SPD for $\sigma \approx 3.98$ (FWHM=3 $\mu \mathrm{m}$ ) on the total energy as a function of clipping factor (for $\lambda_{\mathrm{D}}=532 \mathrm{~nm}$ ).

\section{A. Single wavelength illumination}

Signal-to-noise ratio (SNR) as described in [24] has been used as merit function to evaluate the quality of the output intensity distributions. Light fields at the illumination wavelength $\lambda$, propagated through the original unclipped freeform structures, have been used as reference functions (see $I_{\text {out }}$ at Table 1 ). The quality evaluation shown at Figure 3 reveals that, for single wavelength illumination, only at integer clipping factor values the image signal is much above the noise level. Only at these values light does not interfere destructively to impair the desired intensity distribution. The SNR also increases with increasing $q$, as the number of discontinuities or folds in the surface is reduced, thus more energy lies inside the filter SPD circle of FWHM diameter. At low design wavelengths, since the clipping height $H_{c}$ is directly proportional to $\lambda_{\mathrm{D}}$, a higher number of folds is introduced in the MOF element surface. This explains why the quality at integer $q$ values for $532 \mathrm{~nm}$ is lower than that for $641 \mathrm{~nm}$. Furthermore, a mismatch in the illumination and design wavelengths produces a shift in the quality peaks, that are no more positioned at integer $q$ values. The new position of the local maxima in $q$ can be determined in a different wavelength $\lambda$ by multiplying $q$ by the following factor:

$$
\alpha=\frac{\lambda}{\Delta n(\lambda)} \cdot \frac{\Delta n\left(\lambda_{\mathrm{D}}\right)}{\lambda_{\mathrm{D}}}
$$

Unfortunately, the sampling rate of $q$ used in Figure 3 is not high enough to show all of the shifted peaks of the unmatched blue curve. Figure $4(a-b)$ shows the surface maps for the AT beam shaper and (c-f) some of the output intensity distributions at significant $q$ values.

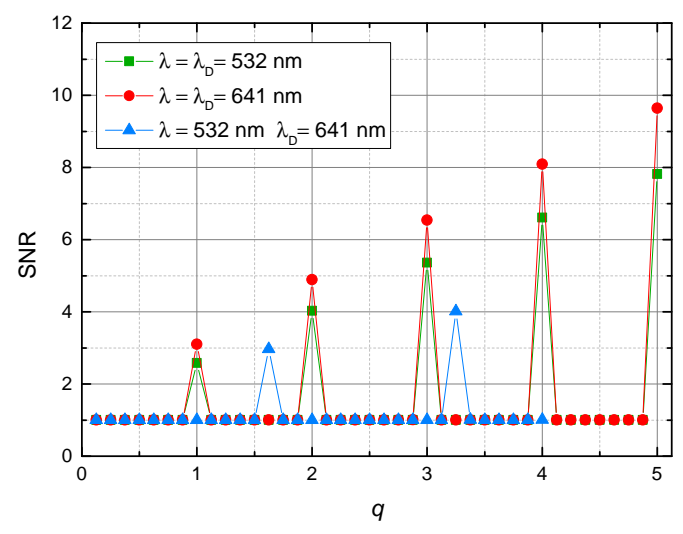

Fig. 3. SNR as function of clipping factor for the AT MOF for monochromatic illumination and different design and illumination wavelengths.

Figure 5 reveals that the MOF element generating the best quality images, with respect to its reference, corresponds to the flattest original freeform element, or the one with less folds or wrinkles for the same clipping factor. Furthermore, in agreement with the curves in Figure 2, it is also the least affected structure by the filtering process. In other words, low-contrast MOF elements generate intensity distributions which are truer to its reference one.

\section{B. Multiple wavelength illumination}

Simulation of broad spectrum illumination has been performed by sampling the spectral power distribution of a standard white LED at five equidistant spectral lines (see Figure 6), and then propagating each light field independently.

In this approach it is assumed that partially coherent light is a superposition of the irradiance from uncorrelated coherent waves. Thereby, the resulting fields are later combined into an RGB image by using the Colorlab Toolbox [25] for Matlab. 


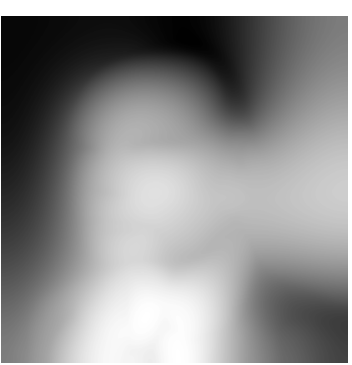

(a)

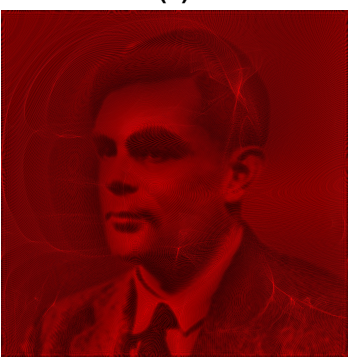

(c)

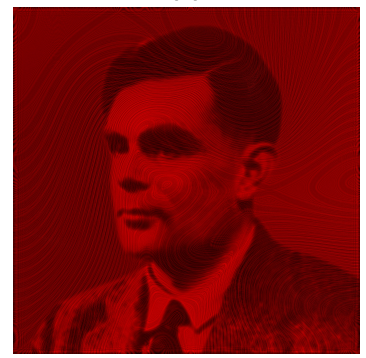

(e)

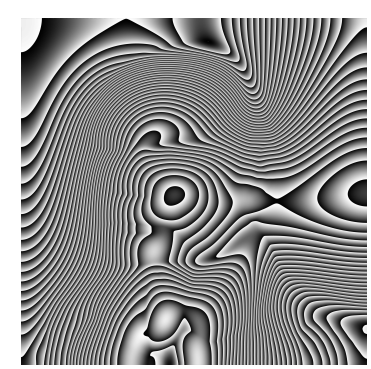

(b)

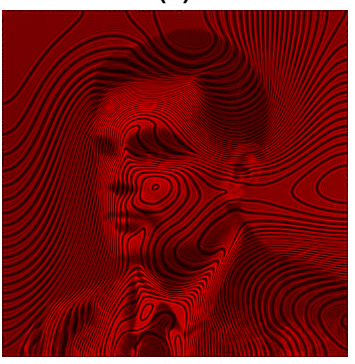

(d)

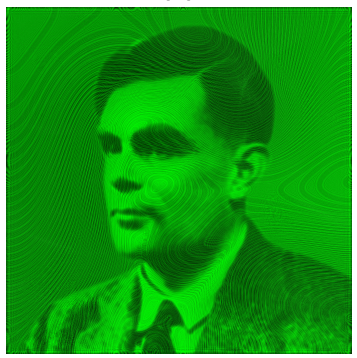

(f)
Fig. 4. Surface map of the (a) scaled original AT freeform element and (b) AT MOF beam shaper $\left(q=5\right.$ for $\left.\lambda_{\mathrm{D}}=641 \mathrm{~nm}\right)$, respectively. Numerical simulation of $I_{\text {out }}$ for illumination at $\lambda=\lambda_{\mathrm{D}}=641 \mathrm{~nm}$ and different clipping factors: (c) $q=1$, (d) $q=4.5$, (e) $q=5$, and (f) $q=3.25$ (illumination at $\lambda=532 \mathrm{~nm}$ ).

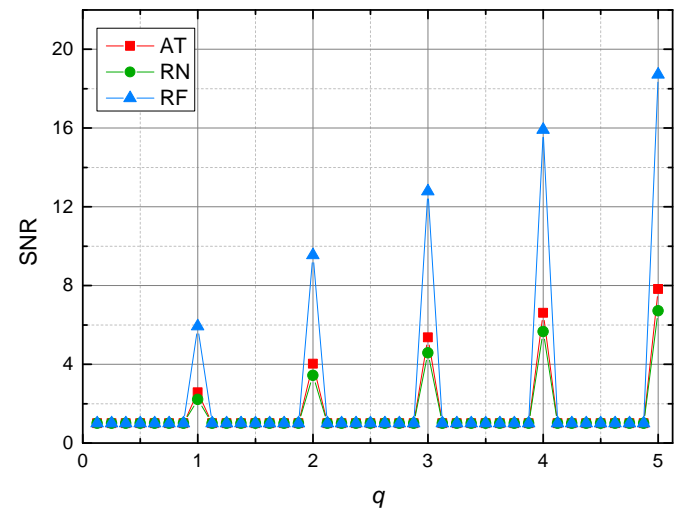

Fig. 5. SNR as function of clipping factor for different MOF elements for monochromatic illumination at $\lambda=\lambda_{D}=532 \mathrm{~nm}$.
Finally, the RGB image has been converted to a grayscale image and its SNR has been computed, as shown in Figure 7. The position of the local maxima in $q$ can be determined in a different design wavelength by dividing $q$ by $\alpha$. A design whose $\lambda_{\mathrm{D}}$ were sitting in the central sample of the illumination spectrum would generate quality local maxima at clipping factors closer to integer values.

So as to avoid incurring into sampling errors for every $\lambda$ and $\lambda_{\mathrm{D}}$ combination up to, at least, $q=5$, the number of samples has been increased to $M=N=25000$, which corresponds to $\Delta x=256 \mathrm{~nm}$ and $\sigma=4.97$ (for the same FWHM filter as in section 3.A). Contrary to the results of Figure 3, the SNR curves upon multiple wavelength illumination do not drop to 1 . This can be explained by the fact that the summation of grayscale intensity distributions coming from color images has been used in the computation of the SNR instead of single complex fields.

It is also worth mentioning that the coherence length of the colorful source is much shorter than that of the monochromatic planar-wave source. Actually, in order to avoid light interferences that could create unwanted artifacts in the output intensity distribution, it would be enough that the coherence length of the source is shorter than the clipping height $H_{c}$ of the MOF element. A rough estimation of the source coherence length gives $2.74 \mu \mathrm{m}$, by using the expression $\langle\lambda\rangle^{2} / \Delta \lambda$.

The reader may have also noted that the SNR gives very similar results at very low $q$ values, e.g. $q=0.125$, and at $q \approx 1$, which could be counter-intuitive. At very low $q$ values, the huge number of folds around the whole MOF surface, in addition to their small height and the filtering process, makes the zeroth order prominent in the multiple wavelength configuration output. If the zeroth order is suppressed for each spectral line, the SNR curve drops to much lower values at very low $q$ values, while it remains comparable at $q \approx 1$ (refer to Figure 8 ). Moreover, the SNR curves do not increase linearly as they did in the case of monochromatic illumination (at integer clipping factors). Indeed, the oscillation amplitude in Figure 7 decreases with $q$ after a certain value, and the expected trend is that the SNR reaches a certain saturation value.

A true color merit function, namely the independent feature similarity (IFS) index [26], has also been evaluated in Figure 7. The $\mid$ IFS $\mid$ curve behaviour fits appropriately that of the SNR curve for $q \geq 1.5$ at $\lambda_{D}=641 \mathrm{~nm}$ and for $q \geq 2.25$ at $\lambda_{D}=532 \mathrm{~nm}$. At lower $q$ values than the ones aforementioned, the SNR curve fits better the subjective quality evaluation than the $|\mathrm{IFS}|$ curve: compare Figure 9 (a) and (b) with their corresponding SNR and |IFS| values at Figure 7.

The IFS index quality evaluation for different MOF elements also differs significantly from the SNR curves for low $q$ values, as showed in Figure 10. The element providing the lowest SNR values, namely $\mathrm{RN}$, is also the one with the highest $|\mathrm{IFS}|$ values of the three elements. Being the element with the highest surface gradients, its folds are the closest to each other. At certain clipping conditions, diffraction effects from folds overlap and give a quite homogeneous output color in relevant regions of the output intensity distribution. These regions are almost free of dark fringes (see RN $I_{\text {out }}$ at Figure 11(e) and compare it with its reference at (a)). The IFS index is a better indicator of the quality perceived by the human vision system than SNR. As the folds separate from one another with increasing $q$, darker fringes appear in the output, and both SNR and |IFS| are impaired (compare RF intensity outputs at Figures 11(d) and (f) and their corresponding quality values at Figure 10). For a $\lambda_{\mathrm{D}}$ centered in the illumination spectrum, the best $|\mathrm{IFS}|$ results 


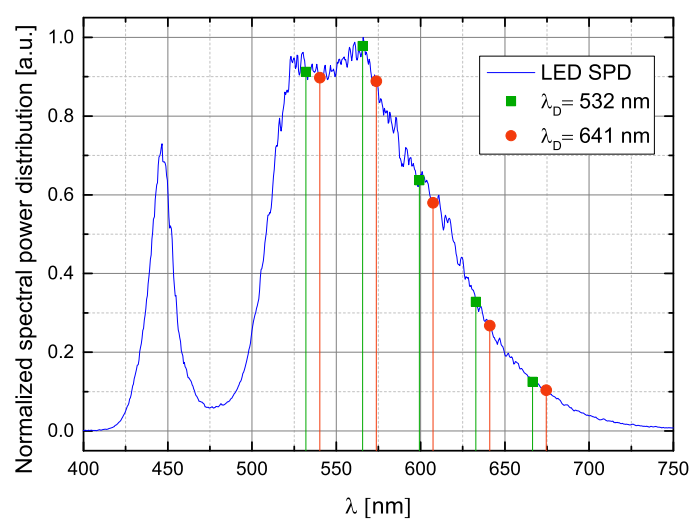

Fig. 6. Normalized spectral power distribution of a standard white LED and the 5 wavelength samples used for each design.

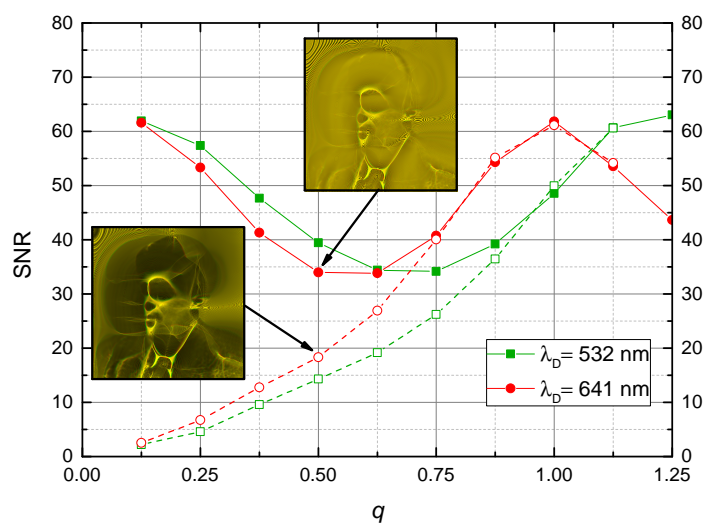

Fig. 8. SNR as function of low clipping factor for different design wavelengths and multiple wavelength illumination of the AT MOF element. Solid symbols and lines represent the normal output and empty symbols and dashed lines show the output with zero-order suppression. The two insets illustrate the output of the standard and the zeroth-order-less curves for $q=0.5$ and $\lambda_{\mathrm{D}}=641 \mathrm{~nm}$.

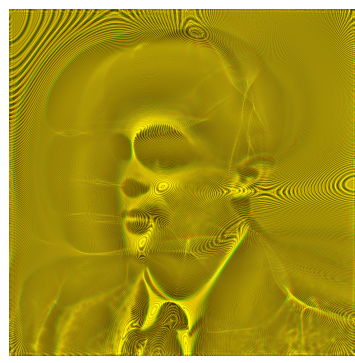

(a)

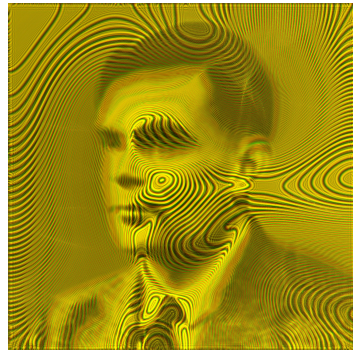

(c)

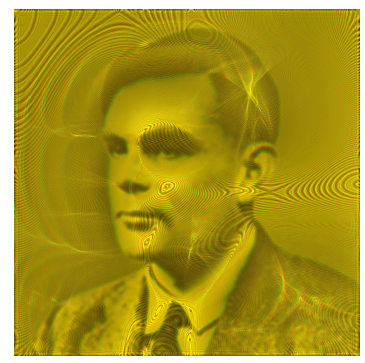

(b)

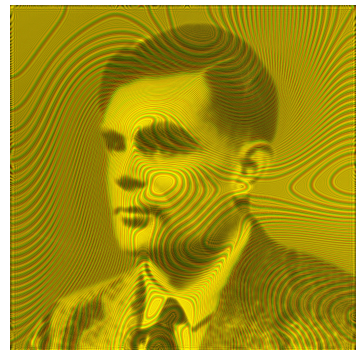

(d)
Fig. 7. SNR (solid symbols and lines) and modulus of the IFS index (|IFS|, empty symbols and dashed lines) as function of clipping factor for different design wavelengths and multiple wavelength illumination of the AT MOF element.
Fig. 9. Numerical simulation of $I_{\text {out }}$ of the AT MOF beam shaper for multiple wavelength illumination at different clipping factors for $\lambda_{\mathrm{D}}=641 \mathrm{~nm}$ : (a) $q=0.625$, (b) $q=1$, (c) $q=2.25$, and (d) $q=3.5$. 


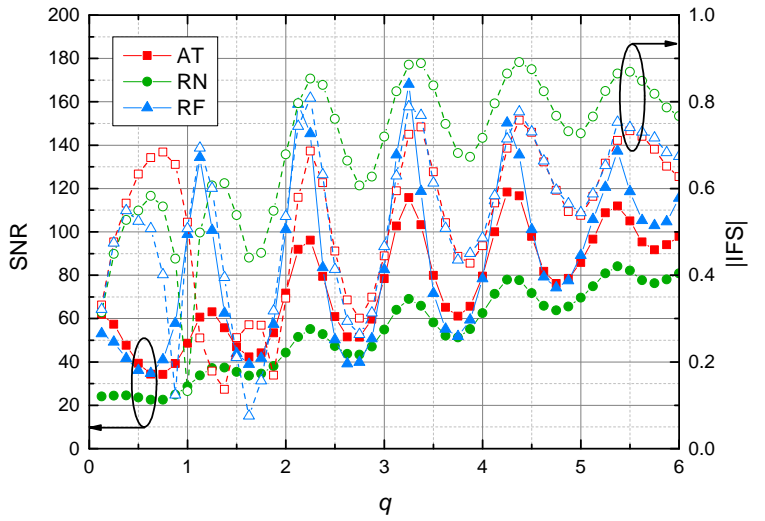

Fig. 10. SNR (solid symbols and lines) and modulus of the IFS index (|IFS|, empty symbols and dashed lines) as function of clipping factor for different MOF beam shapers at $\lambda_{D}=532 \mathrm{~nm}$ and multiple wavelength illumination.

are expected to be obtained at $q \approx 3$. For the $q$ values where the mismatch between $\mid$ IFS $\mid$ and SNR curve shapes is considerable, e.g. $q \lesssim 2$ for AT and $\mathrm{RN}$, and $q \lesssim 1$ for RF, diffraction artifacts are present. Indeed, these effects happen in the region where the height of the structure is smaller than the source coherence length, as $q \lesssim 2.5$.

\section{FABRICATION}

Excimer laser ablation has been used to fabricate the AT MOF element on a polycarbonate substrate foil [27]. Polymer processing allows cost-effective structuring, since the required ablation threshold fluence is not high, as compared to other materials. In the fabrication process used in this work, high energy UV light pulses at $248 \mathrm{~nm}$ wavelength are propagated through a half-tone chromium mask, and then projected by a 0.13 NA objective onto the substrate. Despite the high amount of image artifacts in the intensity distribution at the focus position, see Figure 12(c), this preliminary result is very encouraging for a structure with $P V$ of $11.06 \mu \mathrm{m}$. Further work needs to be conducted in order to reduce the surface roughness (refer to Figure 12(b)) and improve the outcome, as well as the fabrication of structures with different $q$ values.

\section{CONCLUSIONS}

A design method of compact MOF beam shapers has been proposed by wrapping or clipping the original freeform surface profile. An additional low-pass filtering has been applied in order to avoid aliasing effects in light propagation. Monochromatic and multiple wavelength planar-wave illumination have been propagated through the structures and the fidelity of the results with respect to the reference distributions has been evaluated. The number of folds of the MOF beam shaper is directly linked to the $P V$ amplitude of the original freeform element and therefore to the contrast of its output image. In both illumination cases, quality increases as the number of folds is reduced.

Single wavelength illumination reveals that at non-integer clipping factors the quality of the resulting intensity distribution is extremely poor, due to the large coherence length of the light source. In case of a mismatch in the design and illumina-
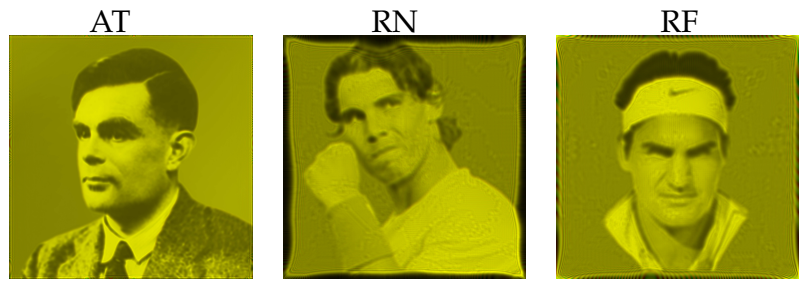

(a) Reference
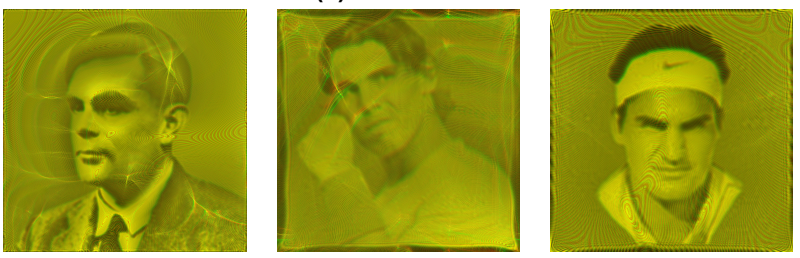

(b) $q=1.125$
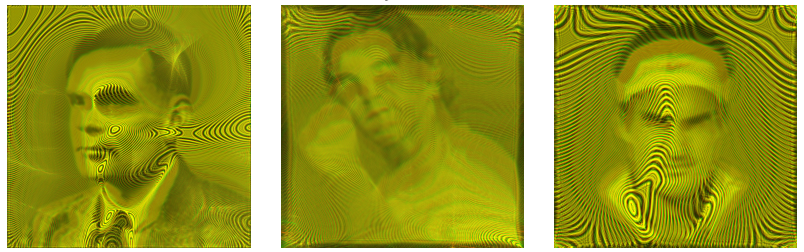

(c) $q=1.625$
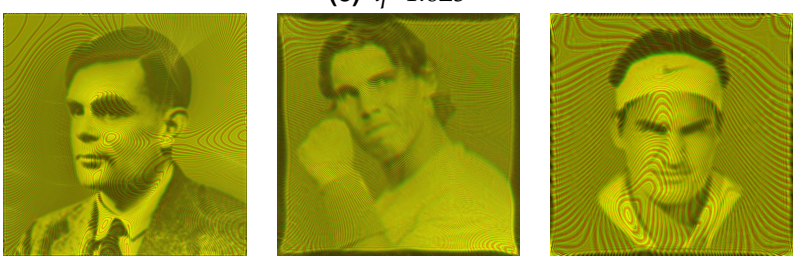

(d) $q=2.25$
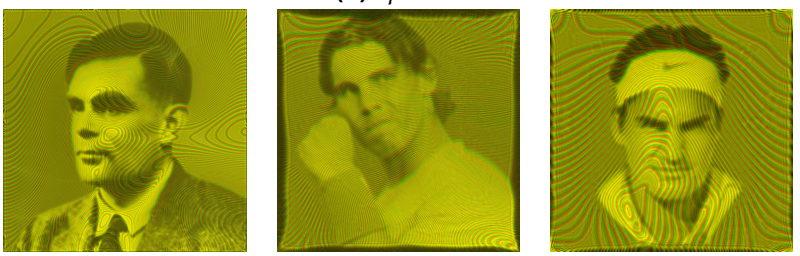

(e) $q=3.25$
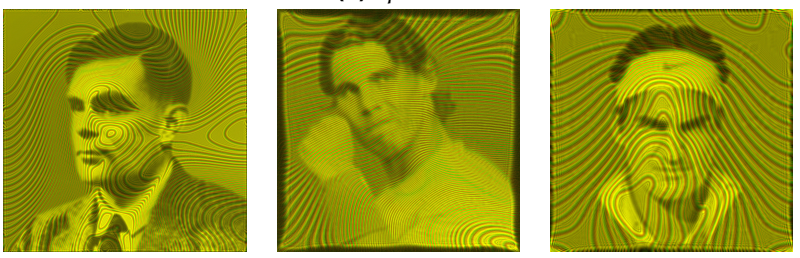

(f) $q=5.375$

Fig. 11. Numerical simulation of $I_{\text {out }}$ of different MOF beam shapers for multiple wavelength illumination at different clipping factors for $\lambda_{\mathrm{D}}=532 \mathrm{~nm}$ : (left) AT, (middle) RN, and (right) RF. 


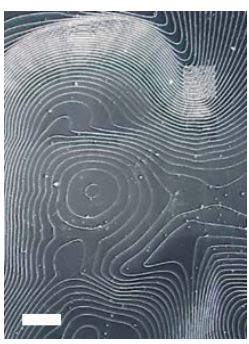

(a)

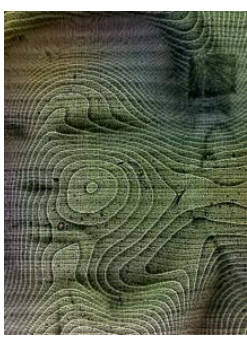

(b)

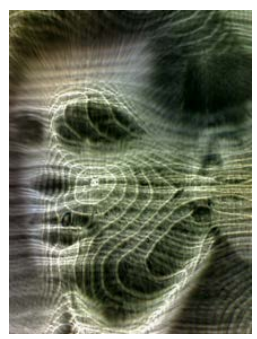

(c)
Fig. 12. (a) Micrograph of the surface of an AT MOF beam shaper $\left(q=10\right.$ for $\left.\lambda_{\mathrm{D}}=641 \mathrm{~nm}\right)$ on polycarbonate, obtained with a 3D digital microscope. The scale bar corresponds to $300 \mathrm{~nm}$. Intensity distribution (b) immediately after the MOF element and (c) at focus position by using a white light source (LED). The images have been cropped due to the limited size of the CCD sensor.

tion wavelengths, the clipping factors providing relatively good image quality are shifted.

In the conditions of broadband illumination, a shorter coherence length of the source than the clipping height $H_{\mathcal{C}}$ allows better quality along a wider range of clipping factors, not only at specific values. Quality increases with $q$ in an oscillatory way but the amplitude of these oscillations tends to decrease. A true color merit function, namely the IFS index, shows different behavior than the SNR for $q \lesssim 1.5$, but is more accurate in representing subjective quality evaluation for the rest of $q$ values. A clipping threshold value for obtaining good quality color intensity distributions is difficult to be set, yet diffraction artifacts become less important when $q \gtrsim 2$. For a design wavelength centered in the illumination spectrum, the best expected quality results correspond to $q \approx 3$. The images obtained from the fabricated sample are promising results for the validation of the simulation work.

\section{FUNDING INFORMATION}

The work here described has been equally funded by the École Polytechnique Fédérale de Lausanne (EPFL) and the Swiss Federal Laboratories for Materials Science and Technology (EMPA).

The authors would like to thank Prof. P. Hoffmann for financial support, Prof. M. Pauly and his laboratory members at EPFL for providing the AT freeform element, and Prof. H. Ries for support with the software ffTOP.

\section{PHOTO CREDITS}

Alan Turing portrait by Elliott \& Fry, (C) National Portrait Gallery, London. Roger Federer portrait courtesy of NIKE, Inc. Rafael Nadal portrait by Matthew Stockman/Getty Images.

\section{REFERENCES}

1. E. Hecht, Optics (Addison-Wesley, Reading, Mass, 2001), 4th ed.

2. A. Lasemi, D. Xue, and P. Gu, "Recent development in CNC machining of freeform surfaces: A state-of-the-art review," Computer-Aided Design 42, 641-654 (2010).

3. R. Brunner, "Transferring diffractive optics from research to commercial applications: Part I - progress in the patent landscape," Advanced Optical Technologies 2, 351-359 (2013).
4. H. Aagedal, M. Schmid, T. Beth, S. Teiwes, and F. Wyrowski, "Theory of speckles in diffractive optics and its application to beam shaping," Journal of Modern Optics 43, 1409-1421 (1996).

5. M. Papas, W. Jarosz, W. Jakob, S. Rusinkiewicz, W. Matusik, and T. Weyrich, "Goal-based Caustics," Computer Graphics Forum 30, 503511 (2011).

6. Y. Schwartzburg, R. Testuz, A. Tagliasacchi, and M. Pauly, "High-contrast Computational Caustic Design," ACM Trans. Graph. 33, 74:1-74:11 (2014).

7. R. Gerchberg and W. Saxton, "Practical algorithm for the determination of phase from image and diffraction plane pictures," Optik (Stuttgart) 35, 237-250 (1972)

8. H. P. Herzig, Micro-Optics: Elements, Systems And Applications (CRC Press, 1997).

9. H. Aagedal, F. Wyrowski, and M. Schmid, "Paraxial beam splitting and shaping," in "Diffractive Optics for Industrial and Commercial Applications," , J. Turunen and F. Wyrowski, eds. (Wiley-VCH, 1998), pp. 165188,1 st ed.

10. F. Sauer, J. Jahns, C. R. Nijander, A. Y. Feldblum, and W. P. Townsend, "Refractive-diffractive micro-optics for permutation interconnects," Opt. Eng 33, 1550-1560 (1994).

11. S. Sinzinger and M. Testorf, "Transition between diffractive and refractive micro-optical components," Appl. Opt. 34, 5970-5976 (1995).

12. T. Hessler, M. Rossi, R. E. Kunz, and M. T. Gale, "Analysis and optimization of fabrication of continuous-relief diffractive optical elements," Appl. Opt. 37, 4069-4079 (1998).

13. T. Nakai and H. Ogawa, "Research on multi-layer diffractive optical elements and their application to camera lenses," in "Diffractive Optics and Micro-Optics," , vol. 75 (OSA, 2002), p. DMA2.

14. J. Salvi, J. Pagès, and J. Batlle, "Pattern codification strategies in structured light systems," Pattern Recognition 37, 827-849 (2004).

15. F. M. Dickey, T. E. Lizotte, S. C. Holswade, and D. L. Shealy, eds., Laser Beam Shaping Applications (CRC Press, Boca Raton, 2005), 1st ed.

16. S. Sinzinger and R. Kleindienst, "Microoptics and freeform optical beam shaping," in "Laser Systems," , H. Weber, P. Loosen, and R. Poprawe, eds. (Springer Berlin Heidelberg, 2011), no. 3 in Landolt-Börnstein Group VIII Advanced Materials and Technologies, pp. 169-193.

17. K. Dholakia and T. Čižmár, "Shaping the future of manipulation," Nat Photon 5, 335-342 (2011).

18. B. Walther, C. Helgert, C. Rockstuhl, F. Setzpfandt, F. Eilenberger, E.-B. Kley, F. Lederer, A. Tünnermann, and T. Pertsch, "Spatial and Spectral Light Shaping with Metamaterials," Adv. Mater. 24, 6300-6304 (2012).

19. J. Dunkel, F. Wippermann, A. Brückner, A. Bräuer, and A. Tünnermann, "Laser lithographic approach to micro-optical freeform elements with extremely large sag heights," Optics Express 20, 4763 (2012).

20. E. C. Harvey, P. T. Rumsby, M. C. Gower, and J. L. Remnant, "Microstructuring by excimer laser," in "Proc. SPIE," , vol. 2639 (1995), pp. 266-277.

21. D. Basting and G. Marowsky, eds., Excimer Laser Technology (Springer, Berlin ; New York, 2005), 2005th ed.

22. D. Infante-Gómez and H. P. Herzig, "Micro-optical freeform elements for beam-shaping," in "Proc. SPIE," , vol. 9630, D. G. Smith, F. Wyrowski, and A. Erdmann, eds. (2015), p. 96300N.

23. J. W. Goodman, Introduction to Fourier Optics (Roberts and Company Publishers, 2005).

24. K. Matsushima, H. Schimmel, and F. Wyrowski, "Fast calculation method for optical diffraction on tilted planes by use of the angular spectrum of plane waves," J. Opt. Soc. Am. A 20, 1755-1762 (2003).

25. "Colorlab Toolbox for Matlab by the University of Joensuu Spectral Color Research Group," http://cs.joensuu.fi/colorlab_toolbox.

26. H.-w. Chang, Q.-w. Zhang, Q.-g. Wu, and Y. Gan, "Perceptual image quality assessment by independent feature detector," Neurocomputing 151, Part 3, 1142-1152 (2015).

27. A. S. Holmes, J. E. A. Pedder, and K. L. Boehlen, "Advanced laser micromachining processes for MEMS and optical applications," in "Proc. SPIE," , vol. 6261 (2006), pp. 62611E-62611E-9. 